\title{
Distance to the nearby dwarf galaxy [TT2009] 25 in the NGC 891 group using the tip of the red giant branch ${ }^{\star}$
}

\author{
Oliver Müller ${ }^{1}$, Rodrigo Ibata ${ }^{1}$, Marina Rejkuba ${ }^{2}$, and Lorenzo Posti ${ }^{1}$ \\ 1 Observatoire Astronomique de Strasbourg (ObAS), Université de Strasbourg - CNRS, UMR 7550, Strasbourg, France \\ e-mail: oliver.muller@astro.unistra.fr \\ 2 European Southern Observatory, Karl-Schwarzschild Strasse 2, 85748 Garching, Germany
}

Received 28 July 2019 / Accepted 8 August 2019

\begin{abstract}
Dwarf galaxies are key objects for small-scale cosmological tests like the abundance problems or the planes-of-satellites problem. A crucial task is therefore to get accurate information for as many nearby dwarf galaxies as possible. Using extremely deep, groundbased $V$ and $i$-band Subaru Suprime Cam photometry with a completeness of $i=27$ mag, we measure the distance of the dwarf galaxy [TT2009] 25 using the tip of the red giant branch as a standard candle. This dwarf resides in the field around the Milky Way-analog NGC 891. Using a Bayesian approach, we measure a distance of $10.28_{-1.73}^{+1.17} \mathrm{Mpc}$, which is consistent with the distance of NGC 891 , and thus confirm it as a member of NGC 891. The dwarf galaxy follows the scaling relations defined by the Local Group dwarfs. We do not find an extended stellar halo around [TT2009] 25. In the small field of view of $100 \mathrm{kpc}$ covered by the survey, only one bright dwarf galaxy and the giant stream are apparent. This is comparable to the Milky Way, where one bright dwarf resides in the same volume, as well as the Sagittarius stream - excluding satellites which are farther away but would be projected in the line-of-sight. It is thus imperative to survey for additional dwarf galaxies in a larger area around NGC 891 to test the abundance of dwarf galaxies and compare this to the number of satellites around the Milky Way.
\end{abstract}

Key words. galaxies: dwarf - galaxies: distances and redshifts - galaxies: groups: individual: NGC 891

\section{Introduction}

In the $\Lambda \mathrm{CDM}$ universe, structure is built up through hierarchical merging in a bottom-up scenario (e.g., the review by Frenk \& White 2012). The smallest building blocks are the dwarf galaxies, which are also the most abundant and the most dark-matterdominated galaxies in the Universe. In recent years, using the dwarf galaxies, several tests of cosmological models have been proposed and conducted, scrutinizing the abundance, the distribution, the motions, and the internal dynamics of the dwarfs (e.g., Moore et al. 1999; Kroupa et al. 2010, 2018a; Boylan-Kolchin et al. 2011; Ibata et al. 2014; López-Corredoira \& Kroupa 2016; Read et al. 2018; Müller et al. 2018a, 2019a; van Dokkum et al. 2018; Javanmardi et al. 2019; Haghi et al. 2019). For some of these tests, the models have successfully found a solution (e.g., Simon \& Geha 2007; Sawala et al. 2016; Kroupa et al. 2018b; Read et al. 2019), while others are still actively discussed in the literature (e.g., Pawlowski et al. 2015; Libeskind et al. 2015; Pawlowski 2018; Müller et al. 2019c; Monelli \& Trujillo 2019). To further improve and extend these tests, it is crucial to measure the above properties for as many dwarf galaxies, and in as many different environments, as possible (Javanmardi et al. 2016; Geha et al. 2017).

In the nearby universe, one of the most effective ways to estimate the distance of a galaxy is via the tip of the red giant

\footnotetext{
* Photometry is only available at the CDS via anonymous ftp to cdsarc.u-strasbg.fr $(130.79 .128 .5)$ or via http://cdsarc. u-strasbg.fr/viz-bin/qcat? J/A+A/629/L2
}

branch (TRGB, Da Costa \& Armandroff 1990; Lee et al. 1993). This method exploits the maximal brightness a low-mass star can reach along its evolutionary path in the RGB phase when observed in appropriate optical or near-infrared (NIR) filters. The observed sharp increase in the (I-band) luminosity function, or cut-off in the color-magnitude diagram (CMD), which is theoretically understood (Serenelli et al. 2017) as well as empirically calibrated (Bellazzini 2008), can be successfully used as a standard candle, yielding distance measurements accurate to about 5 to $10 \%$ (Tully et al. 2015; Müller et al. 2018b; Cohen et al. 2018; Anand et al. 2019; Bennet et al. 2019). In I-band the observed TRGB depends only weakly on age and metallicity, and is typically not affected by the presence of variable stars provided a sufficiently well-sampled stellar population.

In this work, we present a TRGB distance measurement for the dwarf galaxy [TT2009] 25, discovered by Trentham \& Tully (2009) in a wide-field MegaCam-based survey of the region around NGC 1023, and more specifically close to the Milky Way analog NGC 891. This latter galaxy has been targeted to resolve its stellar halo and search for streams through detection of individual RGB stars (Mouhcine et al. 2010) with ground-based Subaru data, accompanied by data from the Hubble space telescope (Rejkuba et al. 2009). While this latter study focused on the stellar halo, which has revealed interaction history with a smaller companion that left streams and loops around NGC 891, in the following we measure the distance to the dwarf galaxy [TT2009] 25, which resides within the deep Subaru field. 


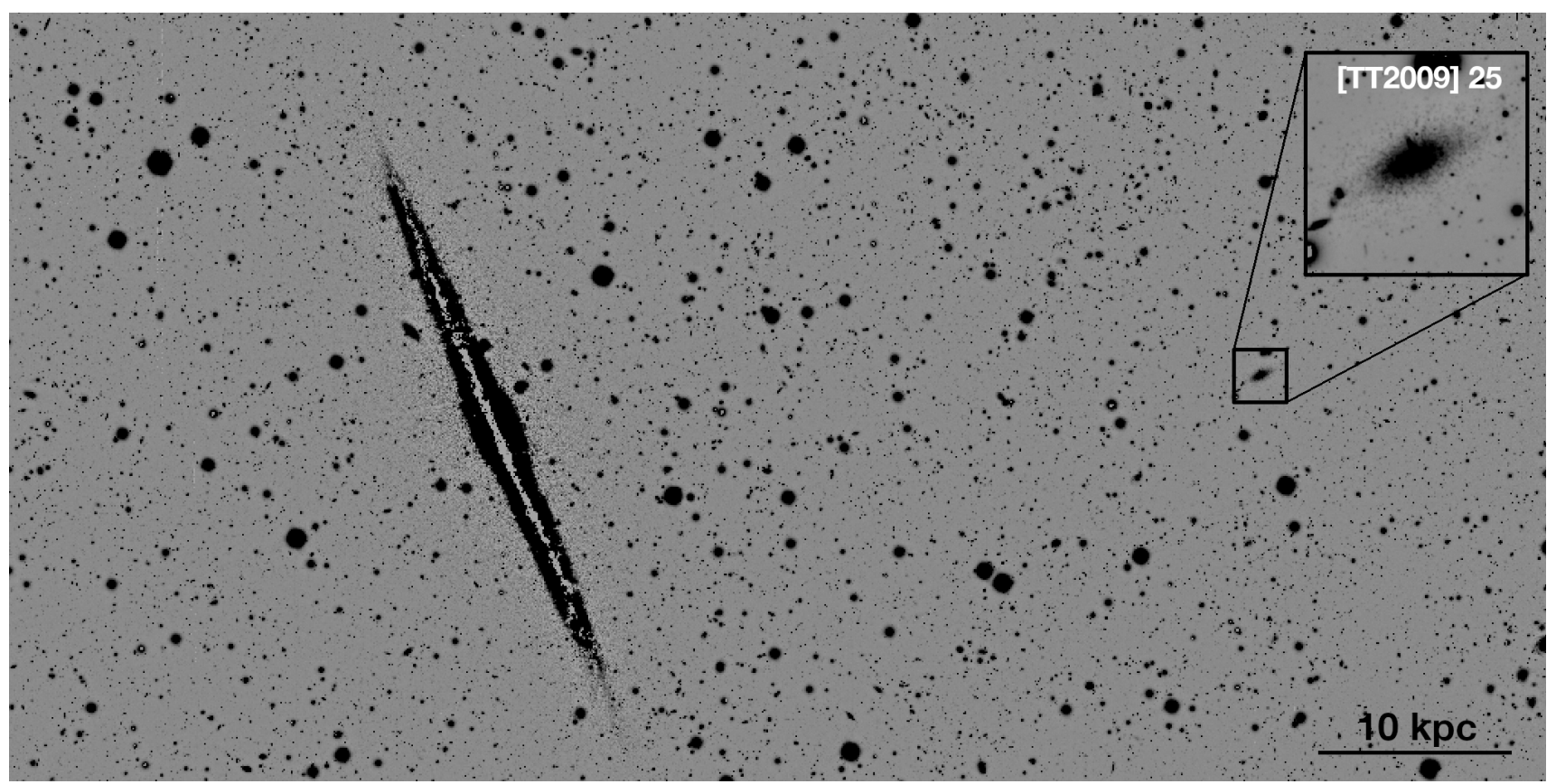

Fig. 1. Field around NGC 891 from our deep, stacked $i$-band Subaru Suprime Cam image. The box highlighting the dwarf galaxy [TT2009] 25 has a side of 1 arcmin. The scale indicates $10 \mathrm{kpc}$ at $10 \mathrm{Mpc}$.

\section{Data and photometry}

In this work we use data from the study of NGC 891 by Mouhcine et al. (2010) based on deep Subaru Suprime Cam (Miyazaki et al. 2002) imaging. Observations were conducted in the Johnson $V$-band and the Gunn $i$-band. A total of $10 \mathrm{~h}$ of good-quality data in the $V$-band and $11 \mathrm{~h}$ in the $i$-band were obtained. The seeing was better than 0.6 arcsec. This strategy resolved the RGB down to 2 mag below the tip at a distance of $10 \mathrm{Mpc}$ (Mouhcine et al. 2007), corresponding to NGC 891.

The photometry was performed with DAOPHOT (Stetson 1987). For the detailed description of the photometric pipeline we refer to Mouhcine et al. (2010). We calibrated the photometry with the Pan-STARRS DR2 catalog (Chambers et al. 2016). While calibrating our $i$-band was a straight forward task, we had to convert Pan-STARRS $g r$ to our $V$ using Lupton (2005). The photometric errors in the $i$-band range from $i \approx 0.05 \mathrm{mag}$ at $i \approx 25.8-26.0 \mathrm{mag}$, that is, the bright end of the RGB, to $i \approx 0.15 \mathrm{mag}$ at $i \approx 26.8-27.0 \mathrm{mag}$ (Mouhcine et al. 2010), i.e., approximately a magnitude below the TRGB of NGC 891. This is sufficient to accurately measure the distance at the 5 to $10 \%$ level. To cull bona-fide stars from background compact galaxies we applied quality cuts using the PSF fitting sharpness and $\chi^{2}$ parameters, and rejected objects deviating from the mean photometric errors (see e.g., Fig. 2 of Müller et al. 2019b).

\section{Distance measurements for the TRGB}

Figure 1 presents a cut-out of the field around NGC 891, including the dwarf. The galaxy is clearly visible and its outskirts are well resolved in stars, while in the center the crowding becomes too high for accurate photometry. Figure 2 shows the star map of putative RGB stars. To create a color magnitude diagram (CMD), we select all stars within an elliptical annulus, avoiding the innermost stars. This was necessary as blending of individual stars was evident, that is, their magnitudes were $\sim 0.4 \mathrm{mag}$ higher than in the outskirts of the galaxy. We refer to Trujillo

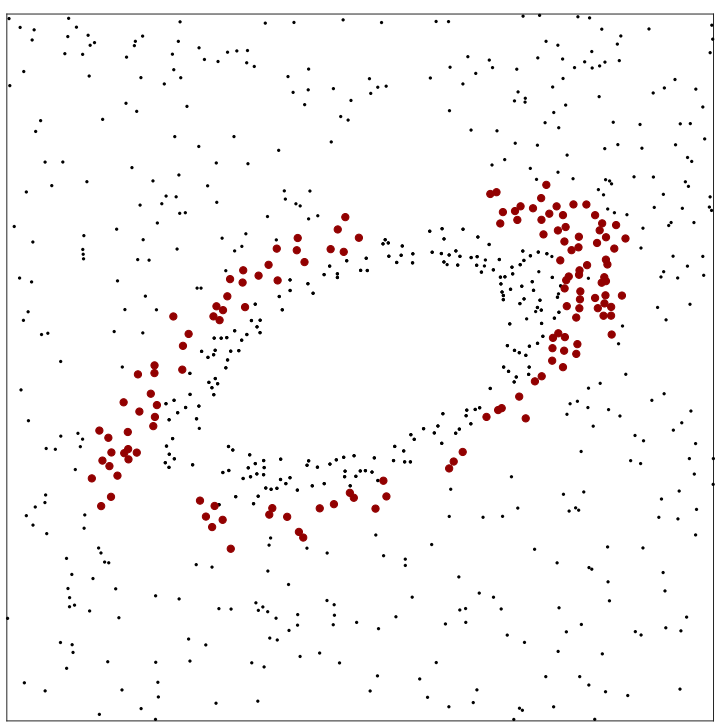

Fig. 2. Star map around [TT2009] 25. Indicated are objects fainter than $i=25.5 \mathrm{mag}$. The large red dots correspond to the stars selected for the CMD. The inner part of the galaxy is obfuscated due to the crowding of stars. One side is 100 arcsec.

et al. (2019) for a recent discussion about the effect of crowding in dwarf galaxies.

We measure the TRGB magnitude using a Bayesian Markov chain Monte Carlo (MCMC) approach developed by Conn et al. (2011, 2012) and described in detail in Müller et al. (2019b). In this scheme, we approximate the location of RGB stars on the CMD with a power law and model the contamination of foreground and background objects using a large reference field. The reference field consists of several patches avoiding the stellar loop and the inner halo of NGC 891, with roughly the same distance to NGC 891 as [TT2009] 25, with an area of $\approx 100$ square arcmin. A most likely set of $m_{\mathrm{TRGB}}, a$, and $c$ are 

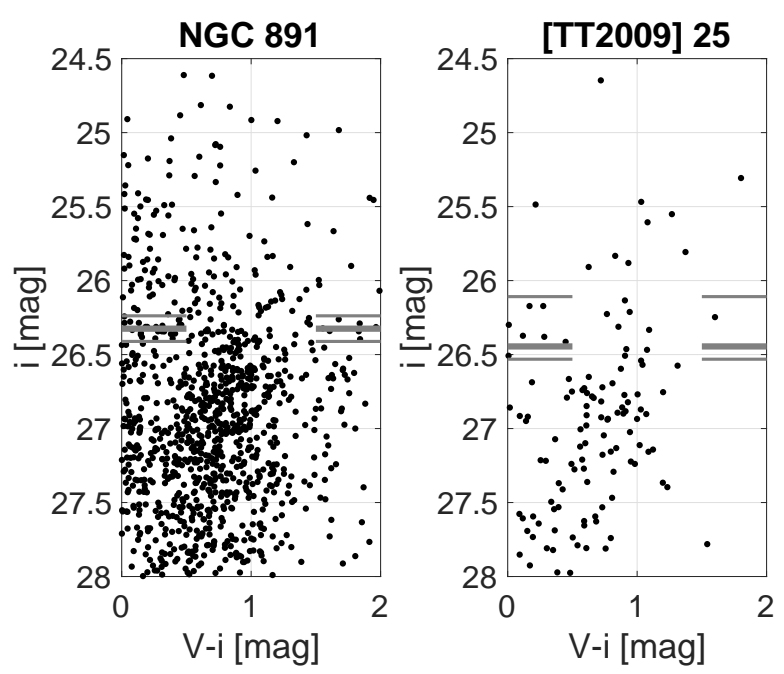

Fig. 3. The CMD of NGC 891 (left) and [TT2009] 25 (right). The gray lines denote the best estimated TRGB magnitude and the corresponding uncertainties.

evaluated, where $m_{\mathrm{TRGB}}$ is the TRGB magnitude, $a$ the slope of the power law, and $c$ the contamination factor.

We estimate the distance to [TT2009] 25 by measuring the difference in the distance modulus between NGC 891 and [TT2009] 25. This carries the advantage that we can anchor our estimate to the distance of NGC 891 coming from the Hubble space telescope, yielding more accurate distances than what is possible from ground-based telescopes due to the superior image quality. For NGC 891, we use an elliptical annulus placed such that it only covers the outskirts of the galaxy. Using our MCMC scheme, we measure $i_{\text {TRGB }}=26.32_{-0.09}^{+0.09}$. The error bounds are given by the $68 \%$ interval of the MCMC chain and already include the photometric uncertainty. This gives a distance of $D=8.97_{-0.36}^{+0.36} \mathrm{Mpc}$ for NGC 891 using the calibration of the TRGB magnitude of $-3.44 \pm 0.1$ (Bellazzini 2008, transformed into $\mathrm{AB}$ magnitudes), which indeed differs slightly from the HST distance of $D=9.73_{-0.86}^{+0.94} \mathrm{Mpc}$ (Mouhcine et al. 2007; Rejkuba et al. 2009). For [TT2009] 25 we measure the TRGB to be at $i_{\text {TRGB }}=26.45_{-0.34}^{+0.09} \mathrm{mag}$. Figure 3 presents the CMD for NGC 891 and the dwarf galaxy [TT2009] 25, as well as the estimated TRGBs. The difference in the distance modulus between NGC 891 and [TT2009] 25 is $0.12 \mathrm{mag}$, however within the uncertainties the two values are consistent. Using a distance of $D=9.73 \mathrm{Mpc}$ for NGC 891 (Mouhcine et al. 2007; Rejkuba et al. 2009) this yields the final extinction corrected distance estimate of $D=10.28_{-1.73}^{+1.17} \mathrm{Mpc}$ for [TT2009] 25, with a distance modulus of $(m-M)=30.06_{-0.40}^{+0.23} \mathrm{mag}$. The uncertainties come from the joint errors of the TRGB detection in the Subaru data $\left({ }_{-0.35}^{+0.12} \mathrm{mag}\right)$ and the uncertainty in the TRGB detection in the HST data $( \pm 0.2 \mathrm{mag})$.

\section{Discussion}

In the following we discuss the structural properties of [TT2009] 25 and the abundance of dwarf galaxy members around NGC 891.

\subsection{Characterizing [TT2009] 25}

The distance estimate of [TT2009] 25 is consistent with it being a satellite of NGC 891. This is not surprising, as the velocity
Table 1. Properties of [TT2009] 25.

\begin{tabular}{|c|c|}
\hline & [TT2009] 25 \\
\hline RA (J2000) & $02: 21: 12.2$ \\
\hline $\operatorname{Dec}(\mathrm{J} 2000)$ & $+42: 21: 50$ \\
\hline$(m-M)_{0}(\mathrm{mag})$ & $30.06_{-0.40}^{+0.23}$ \\
\hline Distance (Mpc) & $10.28_{-1.13}^{+1.17}$ \\
\hline$A_{i}(\mathrm{mag})$ & 0.09 \\
\hline$m_{r}(\mathrm{mag})$ & $16.88 \pm 0.06$ \\
\hline$M_{r}(\mathrm{mag})$ & $-13.18_{-0.40}^{+0.24}$ \\
\hline$L_{r}\left(10^{6} M_{\odot}\right)$ & $16.0_{-3.2}^{+7.1}$ \\
\hline$r_{\mathrm{eff}, r}(\operatorname{arcsec})$ & $13.02 \pm 0.04$ \\
\hline$r_{\mathrm{eff}, r}(\mathrm{pc})$ & $649_{-111}^{+76}$ \\
\hline$\mu_{\mathrm{eff}, r}\left(\operatorname{mag} \operatorname{arcsec}^{-2}\right)$ & $24.45 \pm 0.06$ \\
\hline PA (north to east) & $116.9 \pm 0.1$ \\
\hline$e(1-b / a)$ & $0.52 \pm 0.01$ \\
\hline Sérsic index $n$ & $1.01 \pm 0.01$ \\
\hline
\end{tabular}

$v=692.0 \pm 58 \mathrm{~km} \mathrm{~s}^{-1}$ of [TT2009] 25 (Karachentsev et al. $2015 \mathrm{~b})$ is also consistent with that of NGC $891(v=526 \pm$ $7 \mathrm{~km} \mathrm{~s}^{-1}$, Staveley-Smith \& Davies 1987). Nonetheless, with the distance estimation we confirm beyond doubt that [TT2009] 25 is a dwarf galaxy associated with NGC 891.

Having the distance information for [TT2009]25, we can derive its structural parameters. For this task we use Galfit (Peng et al. 2002) - a standard program to measure galaxy profiles. Due to the dwarf being resolved, we used MegaCam data from the CFHT archive, where the dwarf is unresolved. We downloaded the reduced $r$-band data, calibrated the image using PanSTARRS DR2 (Magnier et al. 2016), and performed aperture photometry with DAOPHOT to measure the zero point of the image. The dwarf galaxy was then fit with a Sérsic model (Sersic 1968) using Galfit. The derived parameters are shown in Table 1.

The structural parameters follow the relation defined by the dwarfs in the Local Group (Martin et al. 2008; McConnachie 2012) and beyond (e.g., Venhola et al. 2017; Müller et al. 2017, 2018c), and are reminiscent of the dwarf spheroidal galaxy Fornax (Irwin \& Hatzidimitriou 1995) in absolute magnitude, effective radius, and ellipticity. The analogy to Fornax goes only so far as [TT2009] 25 has emission in the FUV in GALEX (Martin et al. 2005), indicating star formation. Therefore, [TT2009] 25 is rather a transition-type dwarf than a dwarf spheroidal. Furthermore, no atomic hydrogen is clearly detected with deep radio interferometry at the position of this dwarf galaxy (Oosterloo et al. 2007), which is expected for bound dwarf galaxies around giants (Spekkens et al. 2014).

The stellar profile of [TT2009] 25 seems to have a rather sharp cut-off (visible in Fig. 2). This argues against an extended stellar halo around this dwarf. The existence or absence of such extended stellar halos has been discussed in the literature (e.g., Minniti \& Zijlstra 1996; Rejkuba et al. 2000; Minniti et al. 2003; Gallart et al. 2004; Hidalgo et al. 2009; Greggio et al. 2018), with growing evidence that even low-mass galaxies may have experienced hierarchical growth (e.g., Annibali et al. 2016) and could therefore host extended stellar envelopes. However, the superposition of the outer halo population of NGC 891 in front of [TT2009] 25 would require deeper data to detect any such lowsurface-brightness envelope around the dwarf galaxy (if present).

As a final note, [TT2009] 25 does not seem to be associated with the giant stream around NGC 891. This is evident when studying Fig. 1 of Mouhcine et al. (2010), where the RGB stars 
in the full field are displayed and [TT2009] 25 is well visible as a separated overdensity of stars. The dwarf galaxy seems to be far away from the stream and $\sim 500 \mathrm{kpc}$ behind the halo of NGC 891.

\subsection{Abundance of dwarf galaxies around NGC 891}

Apart from the giant stream surrounding NGC 891, [TT2009] 25 is the only resolved object in our field. This is not unexpected, as the field of view only covers $\sim 100 \mathrm{kpc}$ at the distance of NGC 891. The spiral galaxy NGC 891 is thought to be a Milky Way analog in size and mass. When we take the dwarf galaxy satellites around the Milky Way within a sphere of $50 \mathrm{kpc}$ and brighter than -10 mag (McConnachie 2012; Pawlowski et al. 2013), we find only the Sagittarius dSph and the Large Magellanic Cloud, that is, two satellites. This is quite comparable to what we find for NGC 891: a stream that is reminiscent of Sagittarius and a moderately bright dwarf galaxy. At fainter magnitudes though, the Milky Way hosts at least ten dwarf galaxies (McConnachie 2012), with the numbers still increasing. It is therefore to be expected that many low-mass and low-surface-brightness satellites await discovery within the observed field around NGC 891. Indeed, Ibata et al. (2009) identified six overdensities within the much smaller HST/ACS area imaged close to the main galaxy disk, which could be either candidate satellites or satellite debris. In a wider field of view, Karachentsev et al. (2015a) found no new dwarf galaxy candidates based on a $12 \mathrm{~h}$ exposure with an amateur telescope, apart from [TT2009] 25 and [TT2009]30. This does not mean that NGC 891 is sparsely populated. Within $300 \mathrm{kpc}$, the Local Volume catalog (Karachentsev et al. 2004, 2013) yields four more suspected irregular-type dwarf galaxies based on their radial velocities: DDO 22, DDO 24, UGC 1807, and UGC 2172.

\section{Summary and conclusion}

Using extremely deep $V$ and $i$ band images taken with the Subaru Suprime Cam we resolved the upper part of the RGB of the dwarf galaxy [TT2009] 25. We measured the brightness of the TRGB using a MCMC method and derived an accurate distance for this dwarf galaxy, which is consistent with the distance of NGC 891, thus confirming its membership.

Using data from the CFHT archive, we measured the structural parameters of [TT2009] 25, which are compatible in absolute magnitude, effective radius, and ellipticity with other known dwarf galaxies. Most notably, it is reminiscent of the Local Group dwarf spheroidal galaxy Fornax in morphology, even though the detection of FUV emission makes [TT2009] 25 rather a transitional-type dwarf. Nevertheless, this makes the dwarf galaxy an interesting target for follow-up studies. For example, the globular cluster distribution around Fornax yields strong constraints about the shape of its dark matter halo, favoring a core-like profile over a cusp (Cole et al. 2012; Boldrini et al. 2019; Orkney et al. 2019). Identifying globular clusters around [TT2009] 25 could therefore bring new insight into the cusp/core problem (de Blok 2010), as well as directly measuring the velocity dispersion profile, which should be possible with future facilities like the Thirty Meter Telescope.

Due to the limited field of view, we only cover a region of $50 \mathrm{kpc}$ around NGC 891. In this field, we find one resolved dwarf galaxy. This is comparable to what is expected from the satellite distribution around the Milky Way, where two bright/classical dwarf galaxies reside within a radius of $50 \mathrm{kpc}$. However, this does not include dwarf galaxies which can be farther away from the host, but projected along our line-of-sight, which would increase the number of expected satellites observed around NGC 891. It is therefore imperative to search for dwarf galaxies in a larger area around NGC 891 to test the abundance of dwarf galaxies and compare this to the Milky Way.

Acknowledgements. We thank the referee for the constructive report, which helped to clarify and improve the manuscript. O.M. is grateful to the Swiss National Science Foundation for financial support. The authors thank Filippo Fraternali for discussions about the HI content in the field, and Michele Bellazzini for discussion about the RGB tip calibration in different filter systems.

\section{References}

Anand, G. S., Tully, R. B., Rizzi, L., Shaya, E. J., \& Karachentsev, I. D. 2019, ApJ, 880, 52

Annibali, F., Nipoti, C., Ciotti, L., et al. 2016, ApJ, 826, L27

Bellazzini, M. 2008, Mem. Soc. Astron. It., 79, 440

Bennet, P., Sand, D. J., Crnojević, D., et al. 2019, ApJ, submitted [arXiv:1906.03230]

Boldrini, P., Mohayaee, R., \& Silk, J. 2019, MNRAS, 485, 2546

Boylan-Kolchin, M., Bullock, J. S., \& Kaplinghat, M. 2011, MNRAS, 415, L40

Chambers, K. C., Magnier, E. A., Metcalfe,, N., et al. 2016, ArXiv e-prints [arXiv:1612.05560]

Cohen, Y., van Dokkum, P., Danieli, S., et al. 2018, ApJ, 868, 96

Cole, D. R., Dehnen, W., Read, J. I., \& Wilkinson, M. I. 2012, MNRAS, 426, 601

Conn, A. R., Lewis, G. F., Ibata, R. A., et al. 2011, ApJ, 740, 69

Conn, A. R., Ibata, R. A., Lewis, G. F., et al. 2012, ApJ, 758, 11

Da Costa, G. S., \& Armandroff, T. E. 1990, AJ, 100, 162

de Blok, W. J. G. 2010, Adv. Astron., 2010, 789293

Frenk, C. S., \& White, S. D. M. 2012, Ann. Phys., 524, 507

Gallart, C., Stetson, P. B., Hardy, E., Pont, F., \& Zinn, R. 2004, ApJ, 614, L109

Geha, M., Wechsler, R. H., Mao, Y.-Y., et al. 2017, ApJ, 847, 4

Greggio, L., Falomo, R., \& Scarpa, R. 2018, ApJ, 861, 81

Haghi, H., Kroupa, P., Banik, I., et al. 2019, MNRAS, 487, 2441

Hidalgo, S. L., Aparicio, A., Martínez-Delgado, D., \& Gallart, C. 2009, ApJ, 705,704

Ibata, R., Mouhcine, M., \& Rejkuba, M. 2009, MNRAS, 395, 126

Ibata, N. G., Ibata, R. A., Famaey, B., \& Lewis, G. F. 2014, Nature, 511, 563

Irwin, M., \& Hatzidimitriou, D. 1995, MNRAS, 277, 1354

Javanmardi, B., Martinez-Delgado, D., Kroupa, P., et al. 2016, A\&A, 588, A89

Javanmardi, B., Raouf, M., Khosroshahi, H. G., et al. 2019, ApJ, 870, 50

Karachentsev, I. D., Karachentseva, V. E., Huchtmeier, W. K., \& Makarov, D. I. 2004, AJ, 127, 2031

Karachentsev, I. D., Makarov, D. I., \& Kaisina, E. I. 2013, AJ, 145, 101

Karachentsev, I. D., Riepe, P., Zilch, T., et al. 2015a, Astrophys. Bull., 70, 379

Karachentsev, I. D., Sharina, M. E., Makarov, D. I., Perepelitsyna, Y. A., \&

Safonova, E. S. 2015b, Astrophysics, 58, 309

Kroupa, P., Famaey, B., de Boer, K. S., et al. 2010, A\&A, 523, A32

Kroupa, P., Banik, I., Haghi, H., et al. 2018a, Nat. Astron., 2, 925

Kroupa, P., Haghi, H., Javanmardi, B., et al. 2018b, Nature, 561, E4

Lee, M. G., Freedman, W. L., \& Madore, B. F. 1993, ApJ, 417, 553

Libeskind, N. I., Hoffman, Y., Tully, R. B., et al. 2015, MNRAS, 452, 1052

López-Corredoira, M., \& Kroupa, P. 2016, ApJ, 817, 75

Lupton, R. 2005, Transformations Between SDSS Magnitudes and Other Systems, https://www.sdss3.org/dr10/algorithms/ sdssUBVRITransform.php/

Magnier, E. A., Schlafly, E. F., Finkbeiner, D. P., et al. 2016, ArXiv e-prints [arXiv:1612.05242]

Martin, D. C., Fanson, J., Schiminovich, D., et al. 2005, ApJ, 619, L1

Martin, N. F., de Jong, J. T. A., \& Rix, H.-W. 2008, ApJ, 684, 1075

McConnachie, A. W. 2012, AJ, 144, 4

Minniti, D., \& Zijlstra, A. A. 1996, ApJ, 467, L13

Minniti, D., Borissova, J., Rejkuba, M., et al. 2003, Science, 301, 1508

Miyazaki, S., Komiyama, Y., Sekiguchi, M., et al. 2002, PASJ, 54, 833

Monelli, M., \& Trujillo, I. 2019, ApJ, 880, L11

Moore, B., Ghigna, S., Governato, F., et al. 1999, ApJ, 524, L19

Mouhcine, M., Rejkuba, M., \& Ibata, R. 2007, MNRAS, 381, 873

Mouhcine, M., Ibata, R., \& Rejkuba, M. 2010, ApJ, 714, L12

Müller, O., Jerjen, H., \& Binggeli, B. 2017, A\&A, 597, A7

Müller, O., Pawlowski, M. S., Jerjen, H., \& Lelli, F. 2018a, Science, 359, 534

Müller, O., Rejkuba, M., \& Jerjen, H. 2018b, A\&A, 615, A96

Müller, O., Jerjen, H., \& Binggeli, B. 2018c, A\&A, 615, A105

Müller, O., Famaey, B., \& Zhao, H. 2019a, A\&A, 623, A36 
O. Müller et al.: Distance to the nearby dwarf galaxy [TT2009] 25 in the NGC 891 group using the tip of the red giant branch

Müller, O., Rejkuba, M., Pawlowski, M. S., et al. 2019b, A\&A in press, https://doi.org/10.1051/0004-6361/201935807

Müller, O., Rich, R. M., Román, J., et al. 2019c, A\&A, 624, L6

Oosterloo, T., Fraternali, F., \& Sancisi, R. 2007, AJ, 134, 1019

Orkney, M. D. A., Read, J. I., Petts, J. A., \& Gieles, M. 2019, MNRAS, 1624

Pawlowski, M. S. 2018, Mod. Phys. Lett. A, 33, 1830004

Pawlowski, M. S., Kroupa, P., \& Jerjen, H. 2013, MNRAS, 435, 1928

Pawlowski, M. S., Famaey, B., Merritt, D., \& Kroupa, P. 2015, ApJ, 815, 19

Peng, C. Y., Ho, L. C., Impey, C. D., \& Rix, H.-W. 2002, AJ, 124, 266

Read, J. I., Walker, M. G., \& Steger, P. 2018, MNRAS, 481, 860

Read, J. I., Walker, M. G., \& Steger, P. 2019, MNRAS, 484, 140

Rejkuba, M., Minniti, D., Gregg, M. D., et al. 2000, AJ, 120, 801

Rejkuba, M., Mouhcine, M., \& Ibata, R. 2009, MNRAS, 396, 1231

Sawala, T., Frenk, C. S., Fattahi, A., et al. 2016, MNRAS, 457, 1931
Serenelli, A., Weiss, A., Cassisi, S., Salaris, M., \& Pietrinferni, A. 2017, A\&A, 606, A33

Sersic, J. L. 1968, Atlas de Galaxias Australes (Cordoba, Argentina: Observatorio Astronomico)

Simon, J. D., \& Geha, M. 2007, ApJ, 670, 313

Spekkens, K., Urbancic, N., Mason, B. S., Willman, B., \& Aguirre, J. E. 2014, ApJ, 795, L5

Staveley-Smith, L., \& Davies, R. D. 1987, MNRAS, 224, 953

Stetson, P. B. 1987, PASP, 99, 191

Trentham, N., \& Tully, R. B. 2009, MNRAS, 398, 722

Trujillo, I., Beasley, M. A., Borlaff, A., et al. 2019, MNRAS, 486, 1192

Tully, R. B., Libeskind, N. I., Karachentsev, I. D., et al. 2015, ApJ, 802, L25

van Dokkum, P., Danieli, S., Cohen, Y., et al. 2018, Nature, 555, 629

Venhola, A., Peletier, R., Laurikainen, E., et al. 2017, A\&A, 608, A142 